\title{
Electroweak and QCD corrections to Higgs-boson production in vector-boson fusion at the LHC
}

\author{
Mariano Ciccolini, ${ }^{a}$ Ansgar Denner ${ }^{\star a}$ and Stefan Dittmaier ${ }^{b, c} \dagger$ \\ ${ }^{a}$ Paul Scherrer Institut, Würenlingen und Villigen, CH-5232 Villigen PSI, Switzerland \\ ${ }^{b}$ Max-Planck-Institut für Physik (Werner-Heisenberg-Institut), D-80805 München, Germany \\ ${ }^{c}$ Faculty of Physics, University of Vienna, A-1090 Vienna, Austria \\ E-mail: Mariano.Ciccolini@psi.ch, Ansgar.Denner@psi.ch, \\ Stefan.Dittmaier@mppmu.mpg.de
}

\begin{abstract}
Radiative corrections of strong and electroweak interactions are presented at next-to-leading order for Higgs-boson production in the weak-boson-fusion channel at the LHC. The calculation includes all weak-boson fusion and quark-antiquark annihilation diagrams as well as all related interferences. The electroweak corrections, which also include real corrections from incoming photons and leading heavy-Higgs-boson effects at two-loop order, are of the same size as the QCD corrections, viz. typically at the level of 5-10\% for a Higgs-boson mass up to $\sim 700 \mathrm{GeV}$. In general, they do not simply rescale differential distributions, but induce distortions at the level of $10 \%$. The discussed corrections have been implemented in a flexible Monte Carlo event generator.
\end{abstract}

8th International Symposium on Radiative Corrections (RADCOR)

October 1-5 2007

Florence, Italy

\footnotetext{
* Speaker.

†This work is supported in part by the European Community's Marie-Curie Research Training Network HEPTOOLS under contract MRTN-CT-2006-035505.
} 


\section{Introduction}

The electroweak (EW) production of a Standard Model Higgs boson in association with two hard jets in the forward and backward regions of the detector-frequently quoted as "vector-boson fusion" (VBF)—is a cornerstone in the Higgs search both in the ATLAS [1] and CMS [2] experiments at the LHC and also plays an important role in the determination of Higgs couplings at this collider.

Higgs +2 jets production in pp collisions proceeds through two different channels. The first channel corresponds to a pure EW process. It comprises the scattering of two (anti-)quarks mediated by $t$ - and $u$-channel W- or Z-boson exchange, with the Higgs boson radiated off the virtual weak boson. It also involves Higgs-boson radiation off a W- or Z-boson produced in $s$-channel quark-antiquark annihilation (Higgs-strahlung process), with the weak boson decaying hadronically. The second channel proceeds through strong interactions, the Higgs boson being radiated off a heavy-quark loop that couples to any parton of the incoming hadrons via gluons [3].

In the weak-boson-mediated processes, the two scattered quarks are usually visible as two hard forward jets, in contrast to other jet production mechanisms, offering a good background suppression (transverse-momentum and rapidity cuts on jets, jet rapidity gap, central-jet veto, etc.). Applying appropriate event selection criteria (see e.g. Ref. [4] and references in Refs. [5, 6]) it is possible to sufficiently suppress background and to enhance the VBF channel over the hadronic Higgs +2 jets production mechanism.

In order to match the required precision for theoretical predictions at the LHC, QCD and EW corrections are needed. When VBF cuts are imposed, the cross section can be approximated by the contribution of squared $t$ - and $u$-channel diagrams only, which reduces the QCD corrections to vertex corrections to the weak-boson-quark coupling. Explicit next-to-leading order (NLO) QCD calculations in this approximation exist since more than a decade $[5,7]$, while corrections to distributions have been calculated in the last few years $[8,9]$. Recently, the full NLO EW and QCD corrections to this process have become available $[10,11]$. This calculation includes, for the first time, the complete set of EW and QCD diagrams, namely the $t-, u$, and $s$-channel contributions, as well as all interferences.

In these proceedings we briefly summarize the details of the NLO EW and QCD calculation and give some new results on distributions for a Higgs-boson mass of $200 \mathrm{GeV}$. Numerical results for the Higgs-mass dependence of the total cross section with and without VBF cuts as well as distributions for $M_{\mathrm{H}}=120 \mathrm{GeV}$ have been presented in Refs. [10,11].

\section{Details of the NLO calculation}

We have calculated the complete QCD and EW NLO corrections to Higgs production via weak $\mathrm{VBF}$ at the LHC. At LO, this process receives contributions from the partonic processes $q q \rightarrow \mathrm{H} q q$, $q \bar{q} \rightarrow \mathrm{H} q \bar{q}$, and $\bar{q} \bar{q} \rightarrow \mathrm{H} \bar{q} \bar{q}$. For each relevant configuration of external quark flavours one or two Feynman diagrams contribute in LO. All LO and one-loop NLO diagrams are related by crossing symmetry to the corresponding decay amplitude $\mathrm{H} \rightarrow q \bar{q} q \bar{q}$. The QCD and EW NLO corrections to these decays were discussed in detail in Ref. [12], where a representative set of Feynman diagrams can be found. 
At NLO, there are about $200 \mathrm{EW}$ one-loop diagrams per tree diagram in each flavour channel. The calculation of the one-loop diagrams has been performed in the conventional 't HooftFeynman gauge and in the background-field formalism using the conventions of Refs. [13] and [14], respectively. The masses of the external fermions have been neglected whenever possible, i.e. everywhere but in the mass-singular logarithms. The amplitudes have been generated with FeynArts, using the two independent versions 1 [15] and 3 [16]. The algebraic evaluation has been performed in two completely independent ways. One calculation is based on the in-house Mathematica program that was already used in the algebraic reduction of NLO corrections to the $\mathrm{H} \rightarrow 4$ fermions decays [12]. The other has been completed with the help of FormCalc [17].

In the $s$-channel diagrams intermediate $\mathrm{W}$ and $\mathrm{Z}$ bosons can become resonant, corresponding to $\mathrm{WH} / \mathrm{ZH}$ production with subsequent gauge-boson decay. In order to consistently include these resonances, we use the "complex-mass scheme", which was introduced in Ref. [18] for LO calculations and generalized to the one-loop level in Ref. [19]. In this approach the W- and Z-boson masses are consistently considered as complex quantities, defined as the locations of the propagator poles in the complex plane. The scheme respects all relations that follow from gauge invariance.

The tensor integrals are evaluated as in the calculation of the NLO corrections to $\mathrm{e}^{+} \mathrm{e}^{-} \rightarrow$ 4 fermions $[19,20]$. They are recursively reduced to master integrals at the numerical level. The scalar master integrals are evaluated for complex masses using the methods and results of Ref. [21]. Tensor and scalar 5-point functions are directly expressed in terms of 4-point integrals [22]. Tensor 4-point and 3-point integrals are reduced to scalar integrals with the Passarino-Veltman algorithm [23] as long as no small Gram determinant appears in the reduction. If small Gram determinants occur, the alternative schemes described in Ref. [24] are applied.

Real QCD corrections consist of gluon emission and processes with $g q$ and $g \bar{q}$ initial states. Analogously, real photonic corrections comprise photon bremsstrahlung and photon-induced processes with $\gamma q$ and $\gamma \bar{q}$ initial states. The matrix elements for these corrections have been evaluated using the Weyl-van der Waerden spinor technique as formulated in Ref. [25] and have been checked against results obtained with Madgraph [26]. The phase-space integration is performed using multi-channel Monte Carlo techniques [27] implemented in different ways in two different generators.

All types of real corrections involve singularities from collinear initial-state splittings which are regularized with small quark masses. The mass singularities are absorbed via factorization by the usual PDF redefinition both for the QCD and photonic corrections (see, e.g., Ref. [28]). Technically, the soft and collinear singularities for real gluon or photon emission are isolated both in the dipole subtraction method following Ref. [29] and in the phase-space slicing method. For gluons or photons in the initial state the subtraction and slicing variants described in Ref. [28] are applied. The results presented in the following are obtained with the subtraction method, which numerically performs better.

\section{Numerical results}

We use the input parameters as given in Ref. [11]. Since quark-mixing effects are suppressed, the CKM matrix is set to the unit matrix. The electromagnetic coupling is fixed in the $G_{\mu}$ scheme, i.e. it is set to $\alpha_{G_{\mu}}=\sqrt{2} G_{\mu} M_{\mathrm{W}}^{2} s_{\mathrm{w}}^{2} / \pi$, because this accounts for electromagnetic running effects 


\begin{tabular}{|c|c|c|c|c|c|}
\hline$M_{\mathrm{H}}[\mathrm{GeV}]$ & 120 & 150 & \multicolumn{1}{|c|}{200} & 400 & 700 \\
\hline$\sigma_{\mathrm{LO}}[\mathrm{fb}]$ & $1876.3(5)$ & $1589.8(4)$ & $1221.1(3)$ & $487.31(9)$ & $160.67(2)$ \\
$\sigma_{\mathrm{NLO}}[\mathrm{fb}]$ & $1665(1)$ & $1407.5(8)$ & $1091.3(5)$ & $435.4(2)$ & $160.36(5)$ \\
$\delta_{\mathrm{EW}}[\%]$ & $-6.47(2)$ & $-6.27(2)$ & $-4.98(1)$ & $-3.99(1)$ & $6.99(2)$ \\
$\delta_{\gamma \text {-induced }}[\%]$ & 1.10 & 1.15 & 1.22 & 1.38 & 1.55 \\
$\delta_{\mathrm{QCD}}[\%]$ & $-4.77(4)$ & $-5.20(4)$ & $-5.65(3)$ & $-6.67(3)$ & $-7.18(2)$ \\
\hline
\end{tabular}

Table 1: Cross sections for $\mathrm{pp} \rightarrow \mathrm{H}+2 \mathrm{jets}+X$ in LO and NLO with VBF cuts and relative EW and QCD corrections. The contribution $\delta_{\gamma \text {-induced }}$ from $\gamma$-induced processes (which is part of $\delta_{\mathrm{EW}}$ ) is also given separately.

and some universal corrections of the $\rho$ parameter. We use the MRST2004QED PDF [30] which consistently include $\mathscr{O}(\alpha)$ QED corrections and a photon distribution function for the proton. We use only four quark flavours for the external partons, i.e. we do not take into account the contribution of bottom quarks, which is suppressed. The renormalization and factorization scales are set to $M_{\mathrm{W}}, 5$ flavours are included in the two-loop running of $\alpha_{\mathrm{s}}$, and $\alpha_{\mathrm{s}}\left(M_{\mathrm{Z}}\right)=0.1187$. We apply typical VBF cuts to the outgoing jets as described in detail in Ref. [11].

In Table 1 we present integrated cross sections for $M_{\mathrm{H}}=120,150,200,400$, and $700 \mathrm{GeV}$ for VBF cuts. We list the LO cross section $\sigma_{\mathrm{LO}}$, the cross section $\sigma_{\mathrm{NLO}}$ including QCD+EW corrections, and the relative $\mathrm{QCD}$ and $\mathrm{EW}$ corrections, $\delta_{\mathrm{QCD}}$ and $\delta_{\mathrm{EW}}$, respectively. The complete EW corrections $\delta_{\mathrm{EW}}$ also comprise the corrections from photon-induced processes $\delta_{\gamma \text {-induced }}$, which turn out to be $\sim+1 \%$. The QCD corrections are dominated by the known (vertex-like) corrections to the squared $t$ - and $u$-channel VBF diagrams, while corrections to interference terms are below $0.1 \%$ (see also Ref. [31]).

The EW corrections to distributions for $M_{\mathrm{H}}=200 \mathrm{GeV}$ are qualitatively similar to the distributions for $M_{\mathrm{H}}=120 \mathrm{GeV}$ presented in Ref. [11]. As an example we show the distribution in the transverse momentum $p_{\mathrm{j}_{1}, \mathrm{~T}}$ of the harder tagging jet $\mathrm{j}_{1}$ (jet with highest $p_{\mathrm{T}}$ passing all cuts) in Figure 1. We plot the absolute predictions in LO and in NLO including QCD and EW corrections. In addition, we show the relative QCD and EW corrections separately, as well as their sum. QCD and EW corrections become more and more negative with increasing $p_{\mathrm{j}_{1}, \mathrm{~T}}$. For low transverse momentum these corrections are at the level of $5 \%$, while for $p_{\mathrm{j}_{1}, \mathrm{~T}}=400 \mathrm{GeV}$ they add up to about $-40 \%$. This induces a substantial change in shape of this distribution.

In Figure 2 we present the distribution in the azimuthal angle separation of the two tagging jets. This distribution is particularly sensitive to non-standard contributions to the $\mathrm{H} V V$ vertices [9]. As expected for VBF processes, there is a large azimuthal angle separation between the two tagging jets. While QCD corrections are almost flat in this variable, the QCD+EW corrections exhibit a dependence on $\Delta \phi_{\mathrm{jj}}$ on the level of $7 \%$, which is almost twice as big as for $M_{\mathrm{H}}=120 \mathrm{GeV}$ [11].

\section{Conclusions}

Radiative corrections of strong and electroweak interactions have been discussed at next-toleading order for Higgs production via vector-boson fusion at the LHC. The electroweak corrections affect the cross section by 5\%, and are thus as important as the QCD corrections in this channel. They do not simply rescale distributions but induce distortions at the level of $10 \%$. 

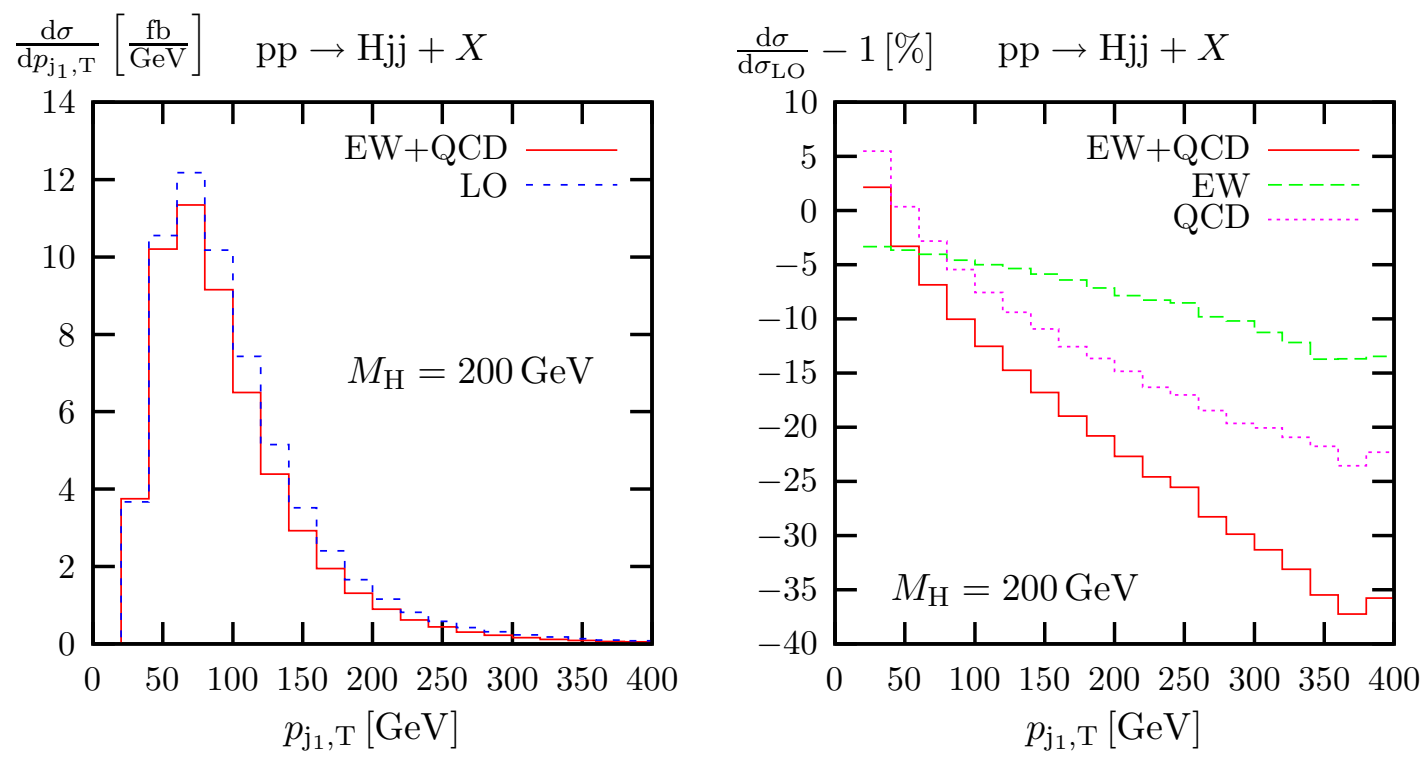

Figure 1: Distribution in the transverse momentum $p_{\mathrm{j}_{1}, \mathrm{~T}}$ of the harder tagging jet (left) and corresponding relative corrections (right) for $M_{\mathrm{H}}=200 \mathrm{GeV}$.
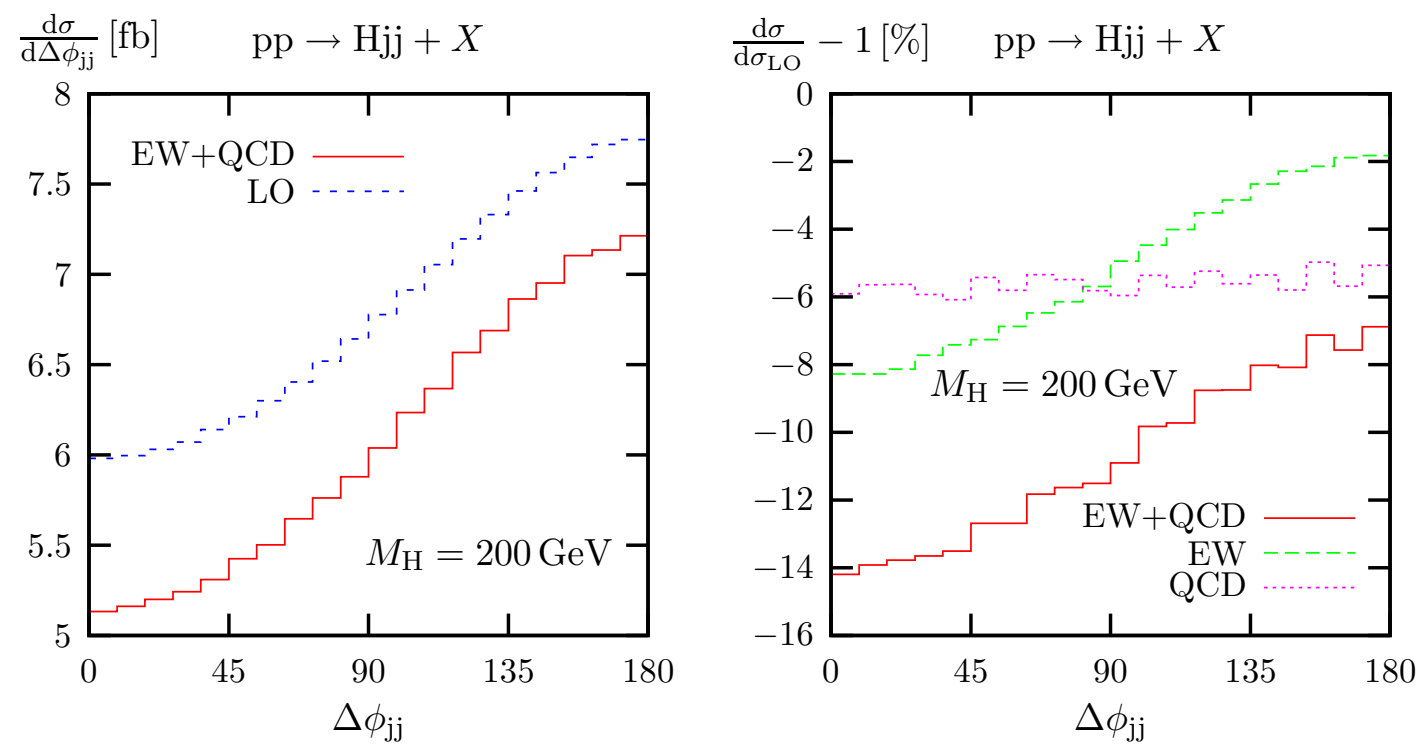

Figure 2: Distribution in the azimuthal angle difference $\Delta \phi_{\mathrm{jj}}$ of the tagging jets (left) and corresponding relative corrections (right) for $M_{\mathrm{H}}=200 \mathrm{GeV}$.

\section{References}

[1] S. Asai et al., Eur. Phys. J. C 32S2 (2004) 19 [hep-ph/0402254].

[2] S. Abdullin et al., Eur. Phys. J. C 39S2 (2005) 41.

[3] V. Del Duca et al., Nucl. Phys. B 616 (2001) 367 [hep-ph/0108030];

J. M. Campbell, R. K. Ellis and G. Zanderighi, JHEP 0610 (2006) 028 [hep-ph/0608194]. 
[4] V. D. Barger, R. J. N. Phillips and D. Zeppenfeld, Phys. Lett. B 346 (1995) 106 [hep-ph/9412276];

D. L. Rainwater and D. Zeppenfeld, JHEP 9712 (1997) 005 [hep-ph/9712271];

D. L. Rainwater, D. Zeppenfeld and K. Hagiwara, Phys. Rev. D 59 (1999) 014037 [hep-ph/9808468];

D. L. Rainwater and D. Zeppenfeld, Phys. Rev. D 60 (1999) 113004 [Erratum-ibid. D 61 (2000) 099901] [hep-ph/9906218];

V. Del Duca et al., JHEP 0610 (2006) 016 [hep-ph/0608158].

[5] M. Spira, Fortsch. Phys. 46 (1998) 203 [hep-ph/9705337].

[6] A. Djouadi, hep-ph/0503172.

[7] T. Han, G. Valencia and S. Willenbrock, Phys. Rev. Lett. 69 (1992) 3274 [hep-ph/9206246].

[8] T. Figy, C. Oleari and D. Zeppenfeld, Phys. Rev. D 68 (2003) 073005 [hep-ph/0306109];

E. L. Berger and J. Campbell, Phys. Rev. D 70 (2004) 073011 [hep-ph/0403194].

[9] T. Figy and D. Zeppenfeld, Phys. Lett. B 591 (2004) 297 [hep-ph/0403297];

[10] M. Ciccolini, A. Denner and S. Dittmaier, Phys. Rev. Lett. 99 (2007) 161803 [arXiv:0707.0381].

[11] M. Ciccolini, A. Denner and S. Dittmaier, arXiv:0710.4749 [hep-ph], to appear in Phys. Rev. D.

[12] A. Bredenstein, A. Denner, S. Dittmaier and M. M. Weber, Phys. Rev. D 74 (2006) 013004 [hep-ph/0604011]; JHEP 0702 (2007) 080 [hep-ph/0611234].

[13] A. Denner, Fortsch. Phys. 41 (1993) 307 [arXiv:0709.1075 [hep-ph]].

[14] A. Denner, S. Dittmaier and G. Weiglein, Nucl. Phys. B 440 (1995) 95 [hep-ph/9410338].

[15] J. Küblbeck, M. Böhm and A. Denner, Comput. Phys. Commun. 60 (1990) 165; H. Eck and

J. Küblbeck, Guide to FeynArts 1.0, University of Würzburg, 1992.

[16] T. Hahn, Comput. Phys. Commun. 140 (2001) 418 [hep-ph/0012260].

[17] T. Hahn and M. Pérez-Victoria, Comput. Phys. Commun. 118 (1999) 153 [hep-ph/9807565];

T. Hahn, Nucl. Phys. Proc. Suppl. 89 (2000) 231 [hep-ph/0005029].

[18] A. Denner, S. Dittmaier, M. Roth and D. Wackeroth, Nucl. Phys. B 560 (1999) 33 [hep-ph/9904472].

[19] A. Denner, S. Dittmaier, M. Roth and L. H. Wieders, Nucl. Phys. B 724 (2005) 247 [hep-ph/0505042].

[20] A. Denner, S. Dittmaier, M. Roth and L. H. Wieders, Phys. Lett. B 612, 223 (2005) [hep-ph/0502063].

[21] G. 't Hooft and M. Veltman, Nucl. Phys. B 153 (1979) 365; W. Beenakker and A. Denner, Nucl. Phys. B 338 (1990) 349; A. Denner, U. Nierste and R. Scharf, Nucl. Phys. B 367 (1991) 637.

[22] A. Denner and S. Dittmaier, Nucl. Phys. B 658 (2003) 175 [hep-ph/0212259].

[23] G. Passarino and M. Veltman, Nucl. Phys. B 160 (1979) 151.

[24] A. Denner and S. Dittmaier, Nucl. Phys. B 734 (2006) 62 [hep-ph/0509141].

[25] S. Dittmaier, Phys. Rev. D 59 (1999) 016007 [hep-ph/9805445].

[26] T. Stelzer and W.F. Long, Comput. Phys. Commun. 81 (1994) 357 [hep-ph/9401258].

[27] J. Hilgart, R. Kleiss and F. Le Diberder, Comput. Phys. Commun. 75 (1993) 191.

[28] K. P. Diener, S. Dittmaier and W. Hollik, Phys. Rev. D 72 (2005) 093002 [hep-ph/0509084].

[29] S. Dittmaier, Nucl. Phys. B 565 (2000) 69 [hep-ph/9904440].

[30] A. D. Martin et al., Eur. Phys. J. C 39 (2005) 155 [hep-ph/0411040].

[31] J. R. Andersen and J. M. Smillie, Phys. Rev. D 75 (2007) 037301 [hep-ph/0611281]; J. R. Andersen, T. Binoth, G. Heinrich and J. M. Smillie, arXiv:0709.3513 [hep-ph]. 